\title{
Erratum: ac-driven annular Josephson junctions: The missing Shapiro steps [Phys. Rev. B 101, 024512 (2020)]
}

\author{
I. R. Rahmonov, J. Tekić $\odot$, P. Mali, A. Irie, and Yu. M. Shukrinov
}

(Q) (Received 27 January 2020; accepted 29 January 2020; published 6 February 2020)

DOI: 10.1103/PhysRevB.101.089901

This manuscript was published online on 21 January 2020, with typographic errors in Eq. (1) and the Acknowledgments. Equation (1) should read

$$
\frac{d^{2} \varphi_{i}}{d t^{2}}-\frac{\varphi_{i+1}-2 \varphi_{i}+\varphi_{i-1}}{a^{2}}+\sin \varphi_{i}+\alpha \frac{d \varphi_{i}}{d t}=I+A \sin (\omega t) .
$$

Since all calculations were performed using the correct equation, this error has no effect on the presented results.

The second sentence in the Acknowledgments should read as follows:

This work was supported by a grant of the fund for developing theoretical physics and mathematics "Basis." 